\title{
Injectable Flexible Subcutaneous Electrode Array Technology for Electrocardiogram Monitoring Device
}

\author{
Jihye Bong ${ }^{1}$, Omar Yasin², Vaibhav R. Vaidya ${ }^{2}$, Jeongpil Park ${ }^{1}$, Zachi I. Attia ${ }^{2}$, Deepak \\ Padmanabhan $^{2}$, Sang June Cho ${ }^{1}$, Roshini Asirvatham ${ }^{2}$, Noah Schneider ${ }^{2}$, Juhwan Lee ${ }^{1}$, Eun Mee \\ $\mathrm{Kim}^{3}$, Paul A. Friedman ${ }^{2, *}$, and Zhenqiang $\mathrm{Ma}^{1, *}$ \\ ${ }^{1}$ Department of Electrical and Computer Engineering, University of Wisconsin-Madison, \\ Madison, WI 53706 USA \\ ${ }^{2}$ Department of Cardiovascular Diseases, Mayo Clinic, Rochester, MN 55905 USA \\ ${ }^{3}$ Department of Emergency Medical Technology, Korea Nazarene University, Cheonan, 31172 \\ South Korea
}

*Corresponding author.

E-mail: friedman.paul@mayo.edu (Paul A. Friedman)

E-mail: mazq@engr.wisc.edu (Zhenqiang Ma)

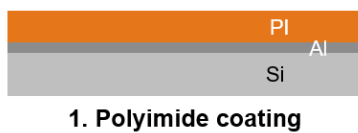

1. Polyimide coating

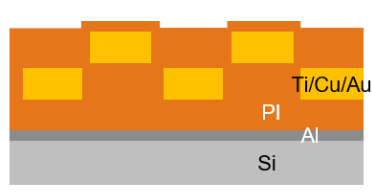

5. $3^{\text {rd }}$ PI layer coating

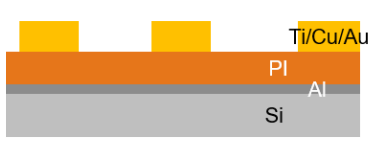

2. Metal patterning by lift-off

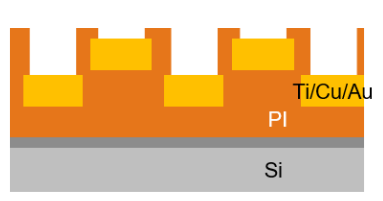

6. PI etching

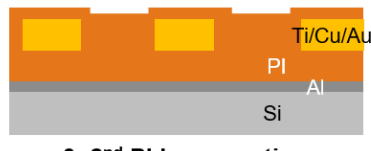

3. $2^{\text {nd }} \mathrm{PI}$ layer coating

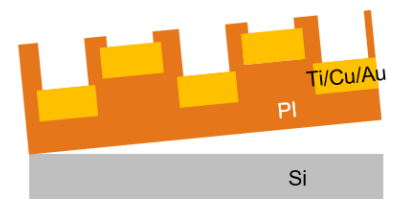

7. PI releasing from substrate

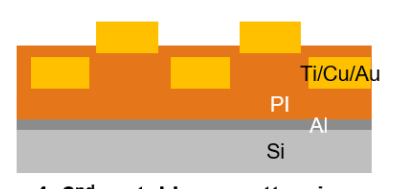

4. $2^{\text {nd }}$ metal layer patterning

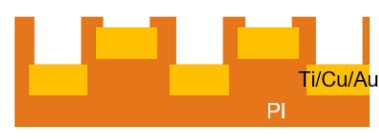

8. Fab-out

Figure S1. Schematic diagram depicting the fabrication process for the injectable electrocardiogram (I-ECG) device. 
a

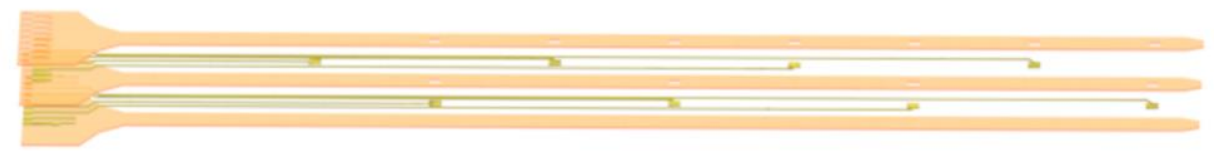

b

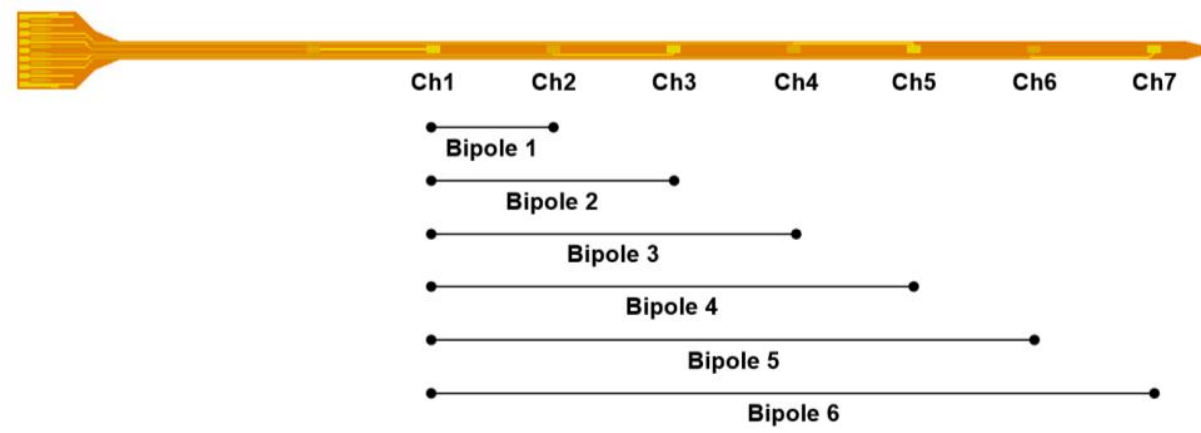

Figure S2. (a) An exploded side and (b) top view of the I-ECG device with number of channel and bipole. Bipole 1 (Ch1-Ch2), Bipole 2 (Ch1-Ch3), Bipole 3 (Ch1-Ch4), Bipole 4 (Ch1-Ch5), Bipole 5 (Ch1-Ch6), and Bipole 6 (Ch1-Ch7).

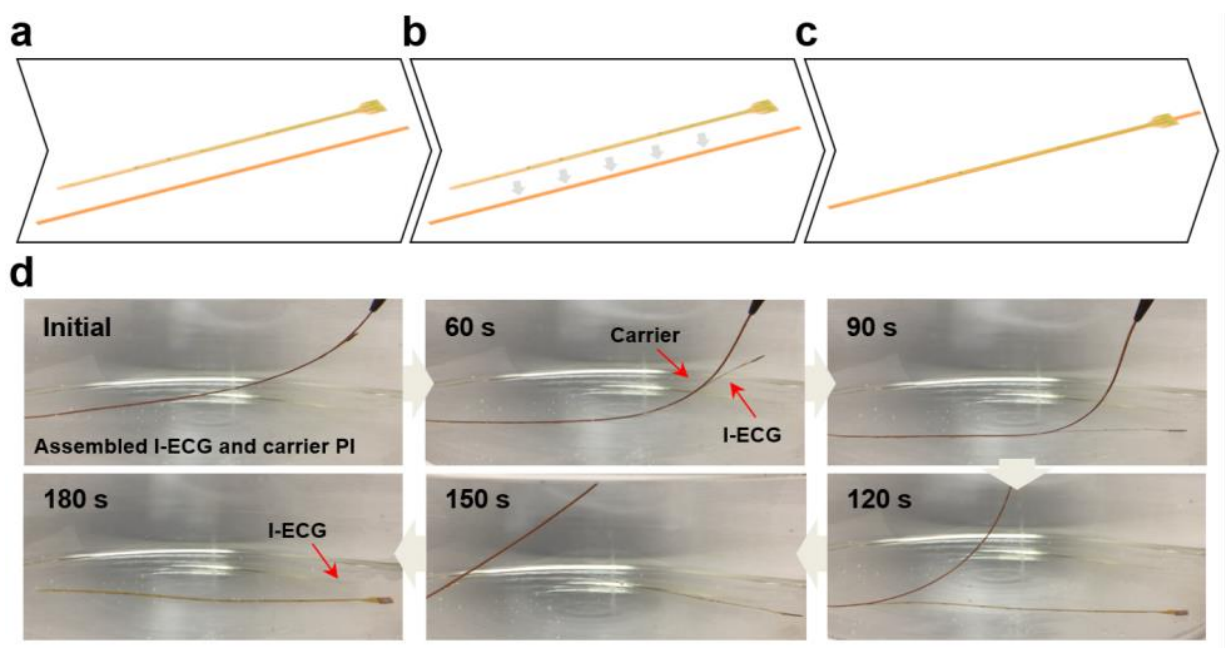

Figure S3. Schematic illustrations to demonstrate the working principle of the procedure. (a) A $500 \mu \mathrm{m}$ thick carrier PI was used for ease of control during the implantation process. (b) PVA is spread between ECG and carrier PI (c) Attached ECG to carrier PI. (d) Sequential photographs of degradation and separation between I-ECG and carrier PI in normal body temperature $\left(37^{\circ} \mathrm{C}\right) \mathrm{DI}$ water. $180 \mathrm{sec}$ is approximately the time constant for this sample.

The PVA was successfully degraded and separation between the carrier PI and I-ECG in normal body was achieved at body temperature $\left(37^{\circ} \mathrm{C}\right)$ DI water (Figure S3d). The assembled I-ECG and carrier PI at initial state was individualized gradually and isolated sequentially by dissolving the PVA. After 3 min, they were completely separated. 
Table S1. ECG parameters in sinus rhythm based on electrodes used to detect the signals. Values reported as mean \pm standard deviations. Each bipole was the average of 20 electro-grams (10 using vertical electrodes and 10 using Horizontal electrodes)

\begin{tabular}{|c|c|c|c|c|c|c|c|c|c|}
\hline Bipole & $\begin{array}{c}\text { RR } \\
\text { interval } \\
(\mathrm{ms})\end{array}$ & $\begin{array}{c}\text { QRS } \\
\text { Amplitude } \\
(\mathrm{mV})\end{array}$ & $\begin{array}{l}\text { QRS } \\
\text { Duration } \\
\text { (ms) }\end{array}$ & $\begin{array}{c}\text { P-wave } \\
\text { Amplitude } \\
(\mathrm{mV})\end{array}$ & $\begin{array}{c}\text { P- wave } \\
\text { Duration } \\
\text { (ms) }\end{array}$ & $\begin{array}{c}\text { PR } \\
\text { Interval } \\
\text { (ms) }\end{array}$ & $\begin{array}{c}\text { T-wave } \\
\text { Amplitude } \\
\text { (mV) }\end{array}$ & $\begin{array}{l}\text { T-wave } \\
\text { Duration } \\
\text { (ms) }\end{array}$ & $\begin{array}{c}\text { QT } \\
\text { Interval } \\
\text { (ms) }\end{array}$ \\
\hline Lead I & $\begin{array}{c}484 \\
( \pm 24.3)\end{array}$ & $\begin{array}{c}0.59 \\
( \pm 0.110)\end{array}$ & $\begin{array}{c}70 \\
( \pm 10.7)\end{array}$ & $\begin{array}{c}0.14 \\
( \pm 0.070)\end{array}$ & $\begin{array}{c}53 \\
( \pm 4.6)\end{array}$ & $\begin{array}{c}108 \\
( \pm 3.7)\end{array}$ & $\begin{array}{c}0.20 \\
( \pm 0.066)\end{array}$ & $\begin{array}{c}75 \\
( \pm 14.2)\end{array}$ & $\begin{array}{c}318 \\
( \pm 7.7)\end{array}$ \\
\hline Lead V1 & $\begin{array}{c}484 \\
( \pm 24.3)\end{array}$ & $\begin{array}{c}0.6 \\
( \pm 0.230) \\
\end{array}$ & $\begin{array}{c}68 \\
( \pm 8.5) \\
\end{array}$ & $\begin{array}{c}0.19 \\
( \pm 0.205)\end{array}$ & $\begin{array}{c}53 \\
( \pm 7.0) \\
\end{array}$ & $\begin{array}{c}110 \\
( \pm 7.6)\end{array}$ & $\begin{array}{c}0.19 \\
( \pm 0.058)\end{array}$ & $\begin{array}{c}73 \\
( \pm 15.2) \\
\end{array}$ & $\begin{array}{c}289 \\
( \pm 29.9)\end{array}$ \\
\hline Lead aVF & $\begin{array}{c}484 \\
( \pm 24.3) \\
\end{array}$ & $\begin{array}{c}0.45 \\
( \pm 0.079)\end{array}$ & $\begin{array}{c}69 \\
( \pm 8.8) \\
\end{array}$ & $\begin{array}{c}0.13 \\
( \pm 0.022) \\
\end{array}$ & $\begin{array}{c}52 \\
( \pm 5.6) \\
\end{array}$ & $\begin{array}{c}122 \\
( \pm 5.4)\end{array}$ & $\begin{array}{c}0.16 \\
( \pm 0.020)\end{array}$ & $\begin{array}{c}74 \\
( \pm 12.7) \\
\end{array}$ & $\begin{array}{c}305 \\
( \pm 35.1)\end{array}$ \\
\hline Bipole 1 & $\begin{array}{c}484 \\
( \pm 24.3)\end{array}$ & $\begin{array}{c}0.14 \\
( \pm 0.047)\end{array}$ & $\begin{array}{c}60 \\
( \pm 10.0)\end{array}$ & $\begin{array}{c}0.02 \\
( \pm 0.019)\end{array}$ & $\begin{array}{c}52 \\
( \pm 8.2)\end{array}$ & $\begin{array}{c}120 \\
( \pm 8.9)\end{array}$ & $\begin{array}{c}0.04 \\
( \pm 0.035)\end{array}$ & $\begin{array}{c}76 \\
( \pm 9.3)\end{array}$ & $\begin{array}{c}308 \\
( \pm 8.6)\end{array}$ \\
\hline Bipole 2 & $\begin{array}{c}478 \\
( \pm 26.1)\end{array}$ & $\begin{array}{c}0.17 \\
( \pm 0.088)\end{array}$ & $\begin{array}{c}71 \\
( \pm 8.8)\end{array}$ & $\begin{array}{c}0.04 \\
( \pm 0.026)\end{array}$ & $\begin{array}{c}55 \\
( \pm 7.5) \\
\end{array}$ & $\begin{array}{c}119 \\
( \pm 8.5)\end{array}$ & $\begin{array}{c}0.09 \\
( \pm 0.023)\end{array}$ & $\begin{array}{c}76 \\
( \pm 8.5) \\
\end{array}$ & $\begin{array}{c}308 \\
( \pm 11.2)\end{array}$ \\
\hline Bipole 3 & $\begin{array}{c}484 \\
( \pm 24.3)\end{array}$ & $\begin{array}{c}0.23 \\
( \pm 0.171)\end{array}$ & $\begin{array}{c}73 \\
( \pm 9.0) \\
\end{array}$ & $\begin{array}{c}0.05 \\
( \pm 0.034)\end{array}$ & $\begin{array}{c}56 \\
( \pm 5.4) \\
\end{array}$ & $\begin{array}{c}121 \\
( \pm 9.1)\end{array}$ & $\begin{array}{c}0.11 \\
( \pm 0.028) \\
\end{array}$ & $\begin{array}{c}78 \\
( \pm 8.3) \\
\end{array}$ & $\begin{array}{c}304 \\
( \pm 9.4)\end{array}$ \\
\hline Bipole 4 & $\begin{array}{c}484 \\
( \pm 24.3)\end{array}$ & $\begin{array}{c}0.29 \\
( \pm 0.112)\end{array}$ & $\begin{array}{c}78 \\
( \pm 12.4)\end{array}$ & $\begin{array}{c}0.07 \\
( \pm 0.014)\end{array}$ & $\begin{array}{c}55 \\
( \pm 7.1)\end{array}$ & $\begin{array}{c}115 \\
( \pm 10.5)\end{array}$ & $\begin{array}{c}0.11 \\
( \pm 0.032)\end{array}$ & $\begin{array}{c}81 \\
( \pm 8.4)\end{array}$ & $\begin{array}{c}305 \\
( \pm 18.6)\end{array}$ \\
\hline Bipole 5 & $497( \pm 3.3)$ & $\begin{array}{c}0.33 \\
( \pm 0.066)\end{array}$ & $\begin{array}{c}80 \\
( \pm 6.2)\end{array}$ & $\begin{array}{c}0.09 \\
( \pm 0.011)\end{array}$ & $\begin{array}{c}58 \\
( \pm 7.4) \\
\end{array}$ & $\begin{array}{c}119 \\
( \pm 9.1)\end{array}$ & $\begin{array}{c}0.12 \\
( \pm 0.031)\end{array}$ & $\begin{array}{c}91 \\
( \pm 11.7)\end{array}$ & $\begin{array}{c}318 \\
( \pm 6.8)\end{array}$ \\
\hline Bipole 6 & $\begin{array}{c}484 \\
( \pm 24.3)\end{array}$ & $\begin{array}{c}0.58 \\
( \pm 0.130)\end{array}$ & $\begin{array}{c}78 \\
( \pm 8.6)\end{array}$ & $\begin{array}{c}0.10 \\
( \pm 0.022)\end{array}$ & $\begin{array}{c}56 \\
( \pm 6.5)\end{array}$ & $\begin{array}{c}119 \\
( \pm 9.7)\end{array}$ & $\begin{array}{c}0.14 \\
( \pm 0.029)\end{array}$ & $\begin{array}{c}85 \\
( \pm 8.8)\end{array}$ & $\begin{array}{c}317 \\
( \pm 10.4)\end{array}$ \\
\hline
\end{tabular}

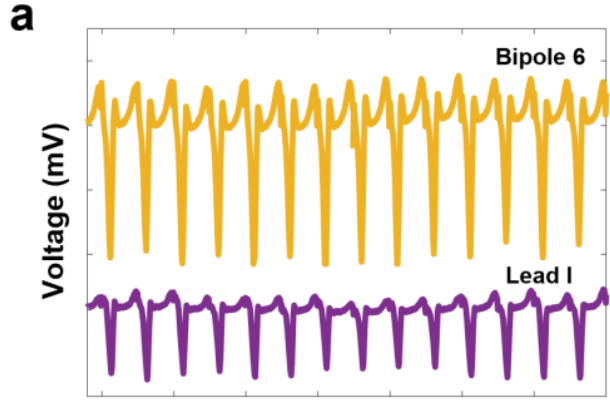

Time (s)

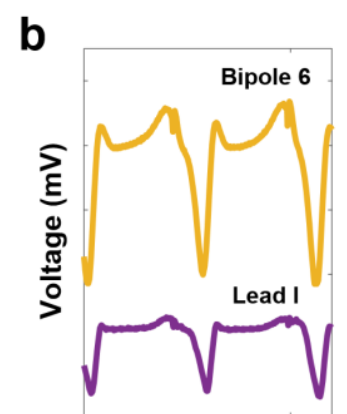

Time (s)

Figure S4. In vivo (a) Simulated arrhythmia (ventricular pacing) signals were detected from the farthest bipolar electrodes and conventional surface ECG. (b) Expanded ECG signals of simulated arrhythmia signals (tick scale: $\mathrm{x}$-axis $0.5 \mathrm{~s}, \mathrm{y}$-axis $0.5 \mathrm{mV}$ ). 